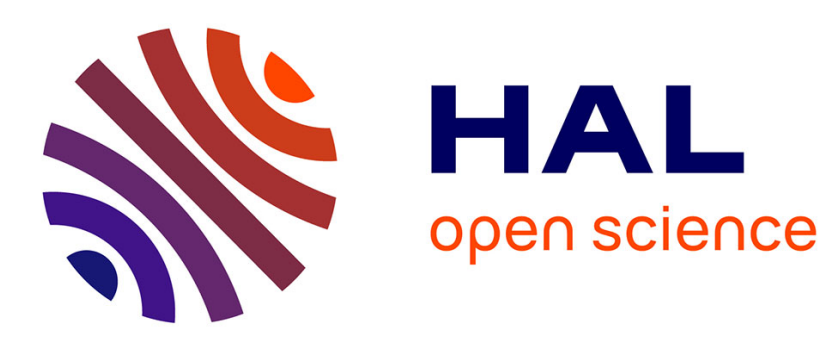

\title{
Chronic interstitial lung diseases in children: diagnosis approaches
}

\author{
Nadia Nathan, Laura Berdah, Keren Borensztajn, Annick Clément
}

\section{To cite this version:}

Nadia Nathan, Laura Berdah, Keren Borensztajn, Annick Clément. Chronic interstitial lung diseases in children: diagnosis approaches. Expert Review of Respiratory Medicine, 2018, 12 (12), pp.10511060. 10.1080/17476348.2018.1538795 . hal-01977067

\section{HAL Id: hal-01977067 https://hal.sorbonne-universite.fr/hal-01977067}

Submitted on 10 Jan 2019

HAL is a multi-disciplinary open access archive for the deposit and dissemination of scientific research documents, whether they are published or not. The documents may come from teaching and research institutions in France or abroad, or from public or private research centers.
L'archive ouverte pluridisciplinaire HAL, est destinée au dépôt et à la diffusion de documents scientifiques de niveau recherche, publiés ou non, émanant des établissements d'enseignement et de recherche français ou étrangers, des laboratoires publics ou privés. 


\title{
Chronic interstitial lung diseases in children: diagnosis approaches
}

\author{
Nadia Nathanª, Laura Berdaha, Keren Borensztajn ${ }^{\mathrm{b}}$ and Annick Clementa,b \\ aService de pneumologie pédiatrique, Centre national de référence des maladies respiratoires rares RespiRare, Hôpital Armand Trousseau, \\ Assistance Publique Hôpitaux de Paris (AP-HP), Paris, France; 'borbonne Université and Inserm UMRS933, Paris, France
}

ABSTRACT

Introduction: Children interstitial lung disease (chILD) is a heterogeneous group of rare respiratory disorders characterized by inflammatory and fibrotic changes of the lung parenchyma. They include ILD related to exposure/environment insults, ILD related to systemic diseases processes, ILD related to primary lung parenchyma dysfunctions and ILD specific to infancy.

Areas covered: This review provides an update on chILD pathophysiology and diagnosis approaches in immunocompetent children. It includes current information on genetic causes. Expert commentary: ChILD covers a large spectrum of entities with heterogeneous disease expression. Various classifications have been reported, but none of them seems completely satisfactory. Recently, progress in molecular genetics has allowed identifying some genetic contributors, with, so far, a lack of correlations between gene disorders and disease expression. Despite improvements in patient manage- ment, chILD prognosis is still burdened by significant morbidity and mortality. Ongoing international collaborations will allow gathering larger longitudinal cohorts of patients to improve disease knowl- edge and personalized care. The overall goal is to help the children with ILD to reach the adulthood transition in a better condition, and to structure genetic counseling for their family.

\section{Introduction}

Interstitial lung disease (ILD) in children is a heterogeneous group of rare respiratory disorders of known and unknown etiologies that are mostly chronic and associated with high morbidity and mortality. ILDs are characterized by inflamma- tory and fibrotic changes of the lung parenchyma structure that typically result in the presence of diffuse infiltrates on lung imaging, and abnormal pulmonary function tests with evidence of a restrictive ventilatory defect and/or impaired gas exchange [1].

\section{Definition and classification}

\subsection{Definition}

The lung interstitium is a support tissue and is composed of three parts: the axial interstitium for the bronchovascular tree, the parenchymal interstitium for the pulmonary parenchyma structure, and the peripheral interstitial for the component adjacent to the pleura [2]. Diseases of the lung interstitium are usually designated as ILD, a term that is not correct as, in most situations, the pathological processes do not affect exclusively the support tissues of the respiratory system. The term of diffuse lung diseases offers a better designation of these disorders, which can involve the alveolar structure as well as the distal part of the small airways and the conducting zone, i.e. the terminal bronchioles. In keeping with the gen- erally disease designation in clinical practice, the term ILD will be used in the present review, with the understanding that it refers to disorders that affect the respiratory function of the lung and consequently the pulmonary structure responsible of the diffusion of gases between blood and air (i.e. the alveolar epithelium, the interstitium, and the pulmonary capillary endothelium) $[3,4]$.

\subsection{Classification}

Many different approaches have been used for the classification of ILD, with major shifts observed over time based on improvements in clinical investigation tools which include chest imaging and close collaboration with pathologists. In adult patients, initial descriptions were histopathological, with proposed 4 distinct subgroups: usual interstitial pneu- monia, desquamative interstitial pneumonia (DIP) and a clo- sely related pattern termed respiratory bronchiolitis- associated ILD, acute interstitial pneumonia (formerly Hamman-Rich syndrome), and nonspecific interstitial pneu- monia (NSIP). Progressively, this historical gold standard has been replaced by a multi-disciplinary approach that has led to define seven specific entities and to provide standardized terminology and diagnostic criteria. This approach resulted in an international multidisciplinary consensus classification of interstitial pneumonias published in 2002 by the American Thoracic Society (ATS)/European Respiratory Society (ERS) that was updated in $2013[5,6]$. The strategies developed for adult ILD classification were similarly used for children ILD (chILD), with initially mainly histologic 
descriptions [7]. Progressively, multi-disciplinary classification schemes that include patient age and clinical situations were set up, providing the basis for clinico-radiologic-pathologic diagnosis $[8,9]$. Patient age is clearly an important issue as the cellular and molecular events associated with the ongoing lung growth and maturation processes are known to interfere with the clinical expression of the pathological disorders [10]. Consequently, the chILD research cooperative group and the European (EU)-chILD collaboration network have individualized the ILD more prevalent in children less than 2 years old, and the ILD not specific to children age [8]. ILD observed mainly during infancy include developmental disorders and growth abnormalities, neuroendocrine cell hyperplasia, and pulmonary interstitial glycogenesis [11,12]. The other pediatric ILD can be grouped into: ILD related to exposure/environment insults, ILD related to systemic diseases processes, ILD related to primary lung parenchyma dysfunctions [1,13-17].

\subsection{Epidemiology}

Estimated prevalence reported in several studies showed large variations that range from 0.1 to 16.2 cases per 100,000 . These numbers are difficult to ascertain and may be under-estimated due to the lack of standardized definitions, the differences in the studied patient populations, and the absence of organized reporting systems. From the limited published data composed mainly of case reports and small series, it seems that pediatric ILD occur more frequently in the younger age and in boys. In addition, nearly $10 \%$ of cases would be familial [18-20].

The present review summarizes current information on ILD diagnosis in immunocompetent children, with a focus on genetic causes.

\section{Clinical features and initial investigations}

\subsection{Clinical presentation of chILD}

The clinical manifestations are often subtle and nonspecific. The onset of symptoms is, in most cases, insidious and many children may have had symptoms for years before the diag- nosis of ILD is confirmed. The clinical manifestations vary from asymptomatic presentation with radiological features sugges- tive of ILD to more characteristic presence of respiratory symptoms and exacerbations [21]. Common symptoms at presentation include dry cough, dyspnea, and chest wall retrac- tion, exercise limitation and frequent respiratory infections. Unexplained fever is also reported, mainly in infants. Failure to thrive, tiring during feeding (in young children) and weight loss are common symptoms in young children $[3,8]$. A history of wheezing may be observed [22]. The frequent clinical find- ings are tachypnea, inspiratory crackles and retraction. Other findings associated with an advanced stage of lung disease include finger clubbing and cyanosis during exercise or at rest. Thorough physical examination includes the search for respira- tory and non-respiratory manifestations such as joint pain, cutaneous rashes and recurrent fever suggestive of underlying systemic diseases. 


\subsection{Chest imaging}

Chest imaging is an important component of disease diagno- sis. Plain radiographs are usually performed in a child sus- pected of ILD at first presentation, but the information provided is often limited and the key tool for diagnosis is the high resolution computed tomography (HRCT), which can visualize the parenchymal structure to the level of the secondary pulmonary lobule. HRCT technique for ILD diagno- sis has been extensively discussed. To optimize spatial resolu- tion, there is a general agreement to use thin sections, the smallest field of view and a sharp resolution algorithm [23]. The most common HRCT feature of ILD is widespread groundglass attenuation. Intralobular lines, irregular interlobular sep- tal thickening and honeycombing are less common findings. Large subpleural air cysts in the upper lobes adjacent to areas of ground-glass opacities have been also reported in young children with ILD. These cysts are interpreted as paraseptal or irregular emphysema. HRCT is useful for ILD diagnosis but is most of the time insufficient to determine the ILD etiology. Consequently, in most situations, diagnosis investigations including molecular studies and lung tissue analysis are warranted. In this context, HRCT is helpful to guide the selection of lung area to be biopsied. It is proposed that it also may contribute to monitor disease progression [24-28]. However, evaluation is still needed to support a role of HRCT as a follow up tool in pediatric patients. Recently, the use of magnetic resonance imaging in the follow-up of pediatric ILD is being discussed, and much effort is currently being engaged to significantly improve image quality [29].

\subsection{Lung function}

Pulmonary function tests do not provide specific information. It may represent a useful investigation tool for both the diag- nosis and the follow-up of ILD in older children and adoles- cents. Generally, in ILD, pulmonary function abnormalities reflect a restrictive ventilatory defect with reduced lung com- pliance and decreased lung volumes. Vital capacity (VC) is variably diminished; the decrease in total lung capacity (TLC) in general is relatively less than in VC. Functional residual capacity (FRC) is also reduced but relatively less than VC and TLC, and residual volume (RV) is generally preserved; thus, the ratios of $\mathrm{FRC/TLC}$ and $\mathrm{RV} / \mathrm{TLC}$ are often increased. Airway involvement is observed only in a minority of patients. Lung diffusing capacity of carbon monoxide (DLCO) or transfer factor (TLCO) is often markedly reduced and may be abnormal before any radiological findings. Hypoxemia as defined by a reduced resting arterial oxygen saturation $\left(\mathrm{SaO}_{2}\right)$ or a reduced resting arterial oxygen tension is often present. Hypercarbia occurs only late in the disease course. During exercise the above described dysfunctions become even more pro- nounced. Thus, gas exchange during exercise might be a more consistent and sensitive indicator of early disease $[1,8]$.

\subsection{Other explorations}

Bronchoscopy with bronchoalveolar lavage (BAL) allows to assess and sample the airways and distal parenchyma. It provides 
specimen for cytological microbiologic and molecular studies; its primary usefulness is to search for infections. It may also orient the diagnosis in situations of alveolar hemorrhage, alveolar proteino- sis, or aspiration with lipid-laden macrophages [30].

Lung biopsy and histological investigations are important investigations, in line with increasing recognition of the different patterns of ILD and their clinical significance. However, it is critical to take into consideration that they are invasive [31]. Consequently, as reported by the European consortium for chILD, histological evaluation of lung tissue usually represents the final step of a series of diagnostic approaches [8]. The methods used to obtain lung tissue, should balance their invasiveness against the potential for obtaining adequate and sufficient tissue for diagnosis. The techniques of choice are open lung biopsy and video assisted thoracoscopy biopsy. The place of other methods such as transbronchial lung biopsy and percutaneous needle lung biopsy remains to be established in pediatric ILD. Samples should be fixed for light microscopy (staining, immunohistochemistry), but also prepared in Glutaraldehyde-buffer for electronic micro- scopy (EM) and frozen for eventual molecular genetics analyses [8,32]. The lung histological patterns which can be observed in pediatric ILD include mainly: DIP, NSIP, and lymphocytic interstitial pneumonia (LIP). DIP is characterized by airspaces filled with alveolar macrophages, thickened alveolar septa, scattered mixed inflammatory cells and minimal fibrosis. Many alveolar spaces are lined by hyperplastic type 2 alveolar epithelial cells (AEC). As example, surfactant disorders have reported to be associated with a DIP pattern on light microscopy and to dense lamellar bodies aspects on EM analysis [33]. NSIP encompasses a broad spectrum of abnormalities with varying degrees of alveolar wall inflammation or fibrosis. The cellular pattern of NSIP is character- ized by mild to moderate interstitial chronic inflammation and type 2 AEC hyperplasia in inflammation areas. It has been reported in a variety of underlying conditions including connective tissues diseases (CTDs) and surfactant disorders. LIP features include a marked diffuse infiltrate of mature lymphocytes, plasma cells and histiocytes within the pulmonary interstitium, particularly the alveolar walls. They are often associated with either CTDs or immunodeficiency states, both congenital and acquired [7,14]. EM analysis can also be of importance in addressing specific diagnoses. As example, glycogen accumulation within the inter- stitial cells has been associated with pulmonary interstitial glycogenosis [34]. Finally, it is of importance also to consider that, depending on disease presentation and suspected diagnosis such as sarcoidosis, tissue biopsy may concern more accessible organs than the lung, such as the skin or the liver.
Based on the clinical context, other investigations could be proposed at patient presentation. They include echocardiogra- phy, mainly for estimation of pulmonary artery pressure if pul- monary hypertension is suspected; blood laboratory tests for evaluation of the immune function, search for autoantibodies, or other biological parameters dictated by the clinical situation.

\section{ILD diagnostic approaches}

A large number of pathological situations can impair gas exchange and contribute to progressive lung damage and ILD. Consequently, diagnosis approaches need to be orga- nized by cause, with a clinical evaluation requiring a careful history paying attention to exposures and systemic diseases. Indeed, from a number of reports it is noteworthy that lung insults caused by substances from the environment or in the context of systemic diseases are largely under-estimated and should be more often considered in the diagnostic process. Importantly, information on relatives or siblings with similar lung conditions are critical and should be thoroughly gath- ered. Accordingly, once the diagnosis of ILD is established on clinical, radiological, and functional findings, a step-bystep etiological diagnostic approach is required [1]. Numerous ILD classifications have been reported. In the present review, we chose to use a clinical approach as diagnostic strategy, with the various pediatric ILD gathered into 4 groups: ILD related to exposure/environment insults, ILD related to systemic diseases processes, ILD related to primary lung parenchyma dysfunc- tions, and ILD specific to infancy (Table 1).

\subsection{ILD related to exposure/environment insults}

They refer to diseases caused by a critical dose of exposure to components with the lung, inducing clinical manifestations and biological impairments. The adult literature has provided exten- sive lists of involved and candidate molecules. In children, less is known about the potential lung involvement of these mole- cules. It is important to stress out that pediatric exposure-related diseases is certainly under-estimated as the diagnosis is less often discussed than in adults [35,36]. Indeed, pediatricians and other child health care providers do not usually have the expertise necessary to retrace an environmental history that has to take into account the home environment but also the day- care, playgrounds, hobbies of the pediatric patient [37].

Hypersensitivity pneumonitis (HP) is a cell-mediated immune reaction to inhaled antigens in susceptible persons.

Table 1. Diagnosis approaches of pediatric ILD.

\begin{tabular}{llll}
\hline $\begin{array}{l}\text { ILD related to exposure/environment } \\
\text { insults }\end{array}$ & $\begin{array}{c}\text { ILD related to systemic diseases } \\
\text { processes }\end{array}$ & $\begin{array}{c}\text { ILD related to lung primary parenchyma } \\
\text { dysfunctions }\end{array}$ & ILD specific to infancy \\
\hline $\begin{array}{l}\text { Hypersensitivity pneumonitis } \\
\text { Medication, Drugs Radiation } \\
\text { exposure }\end{array}$ & Connective tissue diseases & Surfactant disorders & $\begin{array}{c}\text { Neuroendocrine cell hyperplasia of } \\
\text { infancy }\end{array}$ \\
Vasculitis & Diffuse alveolar hemorrhages & Pulmonary interstitial glycogenosis
\end{tabular}

exposure

Granulomatous disorders

Eosinophilic lung diseases

Diffuse developmental disorders 
The responsible antigens have to be searched first in the home environment as well as in relation to certain hobbies. HP are often diagnosed in children at the chronic stage of the disease resulting of a long-term exposure to low levels of inhaled antigens. Children can develop inflammatory reactions in the lung without noticeable symptoms for months. The classical clinical presentation includes non-productive cough, dyspnea, malaise, asthenia and occasional cyanosis [38]. The most frequent types of HP include bird fancier's diseases, humidifier lung diseases, and chemical lung diseases. Bird fancier's diseases, or bird breeder's disease are induced by exposure to birds antigens mainly from avian droppings and feathers $[39,40]$. The disease can occur shortly after the onset of the exposure, or more often after a chronic exposure. Importantly, a suspicion of HP should be raised in case of respiratory symptoms in exposed patients who have only one pet bird at home. Humidifier lung diseases, as well as air conditioner lung, misting fountain lung, basement lung dis- eases and hot tube lung are caused mainly by free-living amoeba and nematodes, as well as bacteria and fungi $[41,42]$. Chemical lung diseases can be induced by various inorganic antigens such as inhaled paints, plastics, wax, talcum etc. [43]. In all cases, once exposure history is suspected, the intervention of an expert environment advisor is mandated to assess the exposure and environmental measurements and biologic tests are necessary to make the causal link between the ILD and the exposure. Serum-precipitating IgG antibodies against the offending antigen will be searched for. However, even if presents, the clinical relevance of the positivity of these antibodies has to be discussed as it is observed in up to $50 \%$ of serum samples of exposed but asymptomatic individuals. Overall, no diagnostic tests are pathognomonic for HP, and only a compatible exposure history and a bundle of biological and radiological signs can be predictive for HP. BAL is neither specific of HP but an alveolitis with a T CD8 lymphocytosis must evoke the diagnosis. Of importance, HP is usually highly sensible to corticosteroids, and a quick and positive response to this treatment, combined with a removal of the causal exposure, can be a supplemental tool for HP diagnosis.

Medications, radiations and drugs can also cause ILD. Drugs used in inflammatory or cancer pediatric diseases can induce ILD. They include anti-inflammatory agents (e.g. aspirin, eta- nercept), immunosuppressive and chemotherapeutic agents (e.g. azathioprine, methotrexate, cyclophosphamide), antibio- tics, cardiovascular agents, and, for teenagers, illicit drugs.

\subsection{ILD related to systemic diseases processes}

They include a number of pathological conditions. CTDs are a heterogeneous group of autoimmune and inflammatory dis- orders that share a common pathophysiology targeting the connective tissues of the body. In childhood, the main CTD disorders involving the lung are rheumatoid arthritis, systemic sclerosis, systemic lupus erythematosus. The other include Sjögren syndrome, dermatomyositis and polymyositis, anky- losing spondylitis, and mixed connective tissue disease [44].
Pulmonary vasculitis affecting small vessels (arterioles, venules, and capillaries) can also be a cause of ILD. They include the anti-neutrophil cytoplasmic antibody (ANCA)associated vas- culitis (Wegener's granulomatosis, ChurgStrauss syndrome, and microscopic polyangiitis); antiglomerular basement membrane (GBM) disease; HenochSchönlein purpura and cryoglobulinemia vasculitis [17]. Recently, genetic forms of autoinflammatory dis- orders involving the lung were also described [45]. Vasculitis can also be diagnosed in situations of diffuse alveolar hemorrhage $(\mathrm{DAH})$. DAH syndromes are caused by the disruption of alveolar- capillary basement membrane as a consequence of injury to the alveolar septal capillaries, and less commonly to the arterioles and venules. The hallmarks are intra-alveolar accumulation of red blood cells, fibrin, and hemosiderin-laden macrophages. It is important to point out that approximately one third of patients with DAH do not manifest hemoptysis, and BAL can be extremely helpful if this entity is suspected by showing the presence of siderophages or red blood cells within the alveoli. In children, situations of DAH in the context of other disorders are reported in several forms of vasculitis discussed above but also in dysim- mune and allergic disorders such as coeliac disease. Vasculitic syndromes that affect large/medium vessels (such as Kawasaki's disease, polyarteritis nodosa) only occasionally affect the lung.

Granulomatous disorders are characterized by the presence of granulomas defined as a focal aggregation of monocellular and giant cells surround by inflammatory cells. Granulomas constitute the result of tissue injury that can be triggered by a variety of agents including microorganisms, antigens, chemical, drugs and other irritants. In other situations, including sarcoi- dosis, the etiologic factors remain to be determined. Sarcoidosis is the most frequent cause of granulomatous ILD. It is a chronic inflammatory disease that is supposed to be the result of a genetic susceptibility, associated with environmental antigens that remain to be identified [46]. The current concept is that a still unknown stimulus activates quiescent $T$ cells and macro- phages leading to recruitment and activation of mononuclear cells, with, as a consequence, granuloma formation, alveolitis, and in some cases interstitial lung fibrosis. The granuloma can develop in many organs, mainly the lung. In children, sarcoido- sis is rare and affects mostly black preteenagers. The diagnosis is based on a combination of suggestive clinical and biological features, with histologically-documented noncaseating granu- loma, in the absence of other known causes of granuloma formation. The disease is often severe in children, presenting as a multi-organic disorder with general symptoms at the forefront (fever, asthenia). Clinical manifestations in sarcoidosis depend on the organ or system involved. Clinical findings mainly include respiratory manifestations, lymphadenopathy, skin lesions, ocular and central nervous system abnormalities. However, pulmonary symptoms can be absent in almost half of the patients with a thoracic sarcoidosis. The most common radiographic findings are hilar lymph node enlargements, with or without ILD. Lung function test can highlight restrictive pulmonary pattern and abnormal diffusing capacity. BAL may document a lymphocytic alveolitis with increased CD4/CD8 ratio, and elevated serum angiotensin-converting 
enzyme may provide additional evidence of sarcoidosis

[47]. 
Other granulomatous diseases in children include infec- tious diseases and Crohn's disease.

Several metabolic disorders can be associated with ILD. Among them, Gaucher's disease, an autosomal recessive dis- ease is the most common lysosomal storage diseases. It is caused by a genetic deficiency of the gluco-cerebrosidase lysosomal enzyme that catalyzes the breakdown of glucocer- ebroside, a cell membrane constituent of red and white blood cells. Niemann-Pick diseases $(A, B, C)$ are rare genetic diseases primarily due to deficiency of sphingomyelinase resulting in the accumulation of sphingomyelin within lysosomes in the macrophage-monocyte phagocyte systems of multiple organs, mainly the brain, spleen, liver, lung, and bone marrow. Histology demonstrates lipid laden macrophages in the mar- row, as well as 'sea-blue histiocytes' on pathology [48]. Hermansky-Pudlak syndrome is a heterogeneous group of autosomal recessive disorders associated with accumulation of a ceroid-like substance in lysosomes of a variety of tissues. It is characterized by albinism, bleeding tendency associated to poor platelet aggregation, and systemic complications asso- ciated to lysosomal dysfunction [49].

\subsection{ILD related to primary lung parenchyma dysfunctions}

This group share common histopathological description, with preserved pulmonary architecture, hyperplasia of type 2 AEC, interstitial infiltrates composed of immune/inflammatory cells and scattered myofibroblasts, and the alveolar space filled with either immuno/inflammatory cells, desquamated materi- als, or components derived from surfactant lipid and protein complex. They include mainly surfactant disorders, $\mathrm{DAH}$, eosi- nophilic lung disease, lymphatic disorders and lung infections. Surfactant disorders are reviewed in the next section.

$\mathrm{DAH}$ can be observed in various clinical situations with or without systemic diseases. In the absence of systemic find- ings, isolated pulmonary capillaritis should be discussed with the search for positivity of the anti-glomerular base- ment membrane antibody with linear deposits in the lung tissue biopsy as well as suggestive serologic features such as p-ANCA antibodies. Cow's milk allergy (Heiner's syn- drome) and diseases induced by environmental factors such as pesticide should also be evocated. Idiopathic pul- monary hemosiderosis is a diagnosis of exclusion based on patient presentation with acute, subacute, or recurrent $\mathrm{DAH}$, on the results of lung biopsy showing evidence of 'bland' pulmonary hemorrhage (ie, without capillaritis or vasculitis), and after exclusion of the conditions listed above. In this situation, red blood cells leak into the alveolar space with- out evidence of damage and/or inflammation of the alveo- lar capillaries [50].

Eosinophilic lung diseases constitute a diverse group of disorders of various origins. The diagnosis is suggested by the presence of pulmonary infiltrates on chest imaging and peripheral eosinophilia. It is confirmed by the presence of increased amounts of eosinophils in $\mathrm{BAL}$ and/or lung tissue eosinophilia. The search for an etiology includes a combina- tion of clinical and laboratory investigations. Eosinophilic lung 
diseases of known cause in children include mainly allergic bronchopulmonary aspergillosis, parasitic infections and drug reactions. Eosinophilic lung diseases of unknown cause com- prise Loeffler syndrome (characterized by migrating pulmon- ary opacities), acute eosinophilic pneumonia, and chronic eosinophilic pneumonia. The idiopathic hyper-eosinophilic syndrome is a rare disorder; it is characterized by prolonged eosinophilia and a multiorgan system dysfunction due to eosinophil infiltration with pulmonary involvement documen- ted in almost half of the patients [51].

Lymphatic disorders can be classified as primary or second- ary. Congenital errors of lymphatic development can lead to primary pulmonary lymphatic disorders that include lymphan- giomas and lymphangiomatosis, lymphangiectasis, and lym- phatic dysplasia syndrome (congenital, yellow nail syndrome). Secondary forms of lung lymphatic disorders result from a variety of processes such as chronic airway inflammation that impair lymph drainage and increase lymph production [52].

The role of infection, mainly viral, in the development and progression of ILD is sustained by a number of human and experimental reports. From recent knowledge, it is strongly suggested that latent viral infections may be involved in the pathogenesis of ILD, through targeting of the alveolar epithe- lium. The main virus implicated include adenovirus, members of herpes virus family human (Epstein-Barrr virus and cytomegalovirus), and respiratory syncitial virus. Number of other viruses can also be involved such as Influenza A or hepatitis $\mathrm{C}$ in immunocompetent children.

\subsection{ILD specific to infancy}

Neuroendocrine cell hyperplasia of infancy (NEHI) is a non- lethal disease characterized by a tachypnea without respira- tory failure in very young infants. The human airway epithe- lium contains highly specialized pulmonary neuroendocrine cells. These cells are the first specialized epithelial cells to appear in the lung. Their function remains unknown but is hypothysed to act as mechano and chemo-sensors and to secrete amines and vasopeptides such as gastrin-releasing peptide (GRP) or bombesin. As normal bombesin level decreases after mid-gestation, its overexpression in NEHI could be attributed to a non-regression of neuroendocrine cells. Some molecular defects have recently been documented [53-55]. Clinical presentation is typically a respiratory distress with tachypnea that usually evolves spontaneously well [56]. Pseudoasthmatic presentations have been reported with wheezing and air-trapping [57]. The lung imaging is character- ized by geographic ground-glass opacifications mainly in the right middle lobe and the lingula. On lung biopsy, the abnor- mal histological findings are minor: hyperplasia of neuroendo- crine cells within terminal bronchioles documented by bombesin immunohistochemistry; light thickening of the air- way smooth muscle; aspecific increase of the alveolar macrophages [58,59].

Pulmonary interstitial glycogenosis (PIG) is also a nonlethal disease of unknown pathogenesis, reported in neonates with respiratory distress syndrome developed shortly after birth. Very few cases have been described so far but it seems to have a male preponderance. The histological hallmark of PIG is 
the accumulation of monoparticulate glycogen in the mesenchymal cells on lung biopsy. It is thought to represent a developmental cell disorder that leads them to accumulate glycogen within their cytoplasm [60]. A histological PIG aspect can be isolated or associated with other pathological condi- tions such as meconium inhalation, other lung growth devel- opment anomalies, and cardiovascular diseases [34]. It is discussed that PIG could meet 'chronic pneumonitis in infancy' as this remains a generalized term.

Diffuse developmental disorders are severe and often lethal conditions [61]. Acinar dysplasia and congenital alveolar dys- plasia are characterized by an arrested development at the pseudoglandular or canalicular/saccular stages, respectively. Alveolar capillary dysplasia with misalignment of pulmonary veins (ACD/MPV) associates an aberrant parenchymal development of the lung with thickened interstitium, a poor capil- lary bed, and in most cases, the presence of pulmonary veins in the bronchovascular axis instead of at the periphery of the lobule. The newborns present with refractory hypoxemia and severe pulmonary hypertension. The diagnosis is usually made on autopsies. Recently, mutations in TBX4 and FGFR2 have been associated with syndromic acinar dysplasia [62,63]. The majority of the described cases of ACD/MPV have been found to be related to FOXF1 mutations or to mutations in the FOXF1 enhancer. Extra-pulmonary disorders are documented in more than half of the cases: congenital heart diseases, digestive malformations or malrotations, and genitourinary malforma- tions [64-66].

Other alveolar growth disorders may be linked to Filamin A defects. Filamin $A$ is a ubiquitous cytoskeletal protein interact- ing with actin. Mutations in FLNA have been associated with dominant syndromic ILD. FLNA is located on the $X$ chromo- some and female have been more described than male [67]. Chest imaging show marked emphysema of the lungs, lobular septal thickening and diffuse patchy atelectasis [68]. In addi- tion to lung manifestations, periventricular nodular heteroto- pias, dental and dermal anomalies, heart diseases and impaired psychomotor development and cognition are reported.

\section{Genetic causes of pediatric ILD}

Genetic factors are important contributors to pediatric ILD. Genetic variations have been mainly described in genes encoding (or interacting with) the surfactant proteins (SP): SP-C (SFTPC) and the ATP-binding cassette-family Amember 3 (ABCA3) (ABCA3), and less frequently in the genes encoding NKX homoeobox 2 (NKX2)-1, (NKX21), SP-B (SFTPB), SP-A

(SFTPA). In situations of pulmonary alveolar proteinosis (PAP), genetic variations in genes encoding the methionyl-tRNA synthetase (MARS) (MARS) and the granulocyte-macrophage colony-stimulating factor (GMCSF) receptors (CSF2RA and CSF2RB) have been reported [69].

\subsection{Genetic disorders of surfactant metabolism}

SFTPC mutations have been associated with various forms of ILD, with heterogeneous clinical presentations from severe neonatal respiratory distress to children and adult ILD and 
lung fibrosis. SP-C is first produced as a pro-protein and then processed through several proteolytic steps to a mature pep-tide of 35 amino acids $(4 \mathrm{kDa})$. The inheritance pattern is autosomal dominant, with incomplete penetrance and sever- ity. Approximately, half of the mutations are de novo muta- tions. To date, more than 40 different mutations have been reported [70-72]. One missense mutation, the c.218T>C (p. lle73Thr) is reported in roughly one third of the cases [73-76]. ABCA3 is a lipidic transporter of the hydrophobic SP-B and SP-C into lamellar bodies. Genetic ABCA3 disorders were first reported in a group of full term babies with severe respiratory diseases occurring shortly after birth and with mostly rapid fatal outcome [77]. It is now known that bi-allelic mutations can also be diagnosed in older children with ILD, and in adult with fibrosing ILD. ABCA3 disorders include mainly hereditary deficiencies in an autosomal recessive inheritance. However, heterozygous $A B C A 3$ mutations have also been associated with an increased risk of neonatal respiratory distress in late preterm newborns. The increasing number of mutations docu- mented in a heterozygous state suggests that $A B C A 3$ defects may be the most common causes of inherited surfactant diseases [78]. To date, more than 200 mutations have been reported in $A B C A 3$, located on chromosome 16, with various heterogeneous clinical expressions, even in siblings [79-81]. One variant, c.875A $>T$ (p.Glu292Val), observed in $0.4 \%$ of the general population, is found in $4 \%$ of a cohort of infants with respiratory distress [82].

Lung disorder resulting from NKX2-1 dysfunction was first reported in an infant with neonatal thyroid disease and respiratory failure [83]. It has since been associated with the 'brain-lung-thyroid' syndrome. The inheritance pattern is auto- somal dominant, with incomplete penetrance and severity for each of the 3 involved organs, even in a single family [84]. Approximately half of the mutations are de novo mutations. NKX2-1 is a transcription factor, located on chromosome 14, that promotes SFTPB, SFTPC and $A B C A 3$ transcription in the alveolar epithelial cells. To date, over 50 mutations have been described, with no recurrent mutation identified [85].

SP-B deficiency was the first surfactant disorder to be described in 1993 in a full-term infant with progressive and fatal respiratory failure [86]. Since this first report, other cases have been reported; most of them being fatal despite aggres- sive treatments and mechanical supports. However, few cases with late survival have also been described. The inheritance pattern is autosomal recessive. SFTPB, is located on human chromosome 2. SP-B is first produced as a proprotein and is then processed through proteolytic cleavages of the $\mathrm{NH} 2$ and $\mathrm{COOH}$ termini to a mature peptide of 79 amino acids $(8 \mathrm{kDa})$. Presently, more than 40 different mutations have been reported, with the frameshift mutation 121ins2, resulting in a premature termination codon, being found in approximately two thirds of cases $[19,87]$.
SP-A the most abundant SP, is a hydrophilic protein that belongs to the C-type lectin family (collectines). The human SP-A locus consists of 2 functional genes: SFTPA1 and SFTPA2, with a pseudo gene (SFTPA3) in between, on chromosome 10. After processing, SP-A1 and SP-A2 assemble to a large multi- meric octadecameric 'bouquet-like' protein structure $[88,89]$. To date, mutations have been mainly documented in SFTPA2 
in adult patients. The phenotype associates various forms of fibrosing ILD, and adenocarcinoma of the lung [90-92]. Recently, our group identified a germline mutation in SFTPA1 in a family with various forms of ILD in an infant, in children and in adults, some of them also presenting with an adeno- carcinoma of the lung. The penetrance was incomplete [93].

\subsection{Other genetic disorders}

MARS deficiencies were first described in PAP by a whole exome sequencing (WES) approach. The patients presented mostly severe and early forms of PAP, some of them being associated with liver and brain involvement [94]. MARS is an ubiquitary enzyme that catalyzes the ligation of methionine to tRNA. It is encoded by MARS, which is located on chromosome

12. Less than 10 bi-allelic mutations have been reported in patients, most of them being originated from La Reunion Island and carrying the same ancestral mutated allele. To date, the relationship between the MARS mutations and the PAP phenotype is not clearly understood.

Very rare cases of pediatric PAP have been associated with GM-CSF receptor defects [95,96]. The clinical presentation is heterogeneous, with patients being diagnosed in adulthood. The effects of GM-CSF are mediated through heterodimeric cell surface receptors that are expressed on a number of cell surfaces including macrophages, neutrophils and type 2 AEC. The GM-CSF receptors are composed of $\alpha$ chains encoded by the CSF2RA gene and $\beta$ chains encoded by the $C S F 2 R B$ gene. It is suggested that the GM-CSF receptor defect could be responsible for a lack of efficiency of the alveolar macro- phages in recycling the SP, that accumulate in the alveolar space.

Other genetic disorders include auto-inflammatory diseases linked to defects in genes encoding the stimulator of inter- feron, STING, also known as transmembrane protein 173 (TMEM173), and the non-clathrin-coated vesicular coat pro- teins (COPs), mainly the Coatomer Protein (COP)- $\alpha$ (COPA) [97]. STING, a stimulator of interferon genes, plays an impor- tant role in innate immunity. It is encoded by TMEM173, located on chromosome 5. Dominant gain-of-function muta- tions of TMEM173 have been associated with an up-regulation of Interferon (IFN) secretion, causing auto-inflammatory and auto-immune disorders [98-100]. The diseases, initially described as 'STING-associated vasculopathie of infancy' (SAVI), have also been reported in adult patients [101]. COP- $\alpha$ is a protein involved in the retrograde transport of the cargo proteins between the golgi and the endoplasmic reticulum. Mutations in COPA, located on chromosome 1 , have recently been described and are associated with childhood onset of multi-organic disorders including ILD, $\mathrm{DAH}$, but also kidney, joint, and immunologic disorders [102-104]. For both STING and COPA disorders, the inheritance pattern is dominant, with an incomplete penetrance of the disease.

\section{Expert commentary}

Pediatric ILD comprises a large spectrum of rare respiratory disorders, some of them being more prevalent in either infants or older children. The age at onset and the disease expression 
are highly heterogeneous. Several classifications have been proposed so far based on histological descriptions and patient presentations. However, none of them appears to be comple- tely satisfactory. As such, surfactant molecular disorders have been recently described in NEHI patients. The development of molecular diagnosis, especially with the generalization of next generation sequencing, allows to document genetic disorders in an increasing number of chILD [105]. In the next years, the classifications will include these novel findings on underlying molecular mechanisms. Along with a better description of the pathologies, there is an urgent need for more specific treat- ments and care. To date, the therapeutic managements of most pediatric ILD remain limited and are mainly based of the use of corticosteroids. However, their efficacy is highly variable, most likely due to the vast heterogeneity of the diseases. New therapeutical strategies targeting specific mole- cular mechanisms are in progress. They include the use of tyrosine kinase inhibitor or transforming growth factor inhibi- tor in children. In addition, lung transplantation is being devel- oped for pediatric patients. So far, pediatric lung transplantation has been reported in a limited number of patients with surfactant disorders (SFTPB, SFTPC, ABCA3), SAVI and COPA syndrome, and in a few cases of developmental disorders, but it remains an exceptional procedure available in a small number of countries [106,107].

\section{Five-year view}

A better understanding of the pathophysiological mechanisms and the natural course of the diseases is a crucial issue. The rarity of the diseases makes this goal a challenge that deserves international clinical and scientific collaborations to gather large longitudinal cohorts of patients, and to develop ongoing basic research. Recently, a European research group of clini- cians and researchers has been launched by the European Cooperation in Science and Technology (COST) action (ENTeR-chILD - CA-1625) to develop multidisciplinary approaches on clinical issues and to explore the various pre- clinical models of ILD. It will provide new insights into the molecular/environmental basis of ILD pathogenesis (including genetic factors causing familial diseases) in children. As such, it is expected that newly identified molecular defects and mar- kers will help predicting clinical courses and tailoring indivi- dual therapies.

\section{Key issues}

- Interstitial lung disease (ILD) is a heterogeneous group of rare respiratory disorders that include ILD related to expo- sure/environment insults, ILD related to systemic diseases processes, ILD related to primary lung parenchyma dysfunc- tions, and ILD specific to infancy.

- Current chILD classifications remain unsatisfactory, due to the complexity of the underling mechanisms and the varia- bility of the disease expression.

- ChILD are mostly severe diseases with high morbidity and mortality.

- Management strategies and therapeutic options remain limited. 
- Genetic factors are important contributors to chILD patho-

genesis, and it is anticipated that genetic testing will have an important role in chILD clinical practice in the next years. Surfactant disorders play important roles in the develop- ment of various forms of chILD. In addition to surfactant genes, other disease-causing genes are increasingly impli- cated in chILD pathogenesis.

- chILD phenotypic expression is highly variable and, cur-

rently, no correlations between disease presentation and molecular defect could be documented.

- International networking is in progress to help deciphering

the chILD molecular mechanisms, predicting disease courses and improving patient management.

\section{Acknowledgments}

The authors would like to thank the Assistance Publique Hôpitaux de Paris, Sorbonne Université, Paris, France, and the national networks for rare lung diseases: Centre de référence des maladies respiratoires rares (RespiRare), Centre de référence des maladies pulmonaires rares (OphaLung) and Filière de soins pour les maladies respiratoires rares (RespiFIL). The ILD cohort is developed in collaboration with the Rare Cohort Disease (RaDiCo)-ILD project (ANR-10-COHO-0003), the FP7- 305653-child-EU project and the COST Action European network for translational research in children's and adult interstitial lung disease pro- ject (ENTeR-chILD - CA16125).

\section{Funding}

This work was supported by the Institut National de la Santé et la Recherche Médicale (INSERM); the Legs Poix from the Chancellerie des Universités, Paris [grants 2013 to 2017 number 1305, 1405, 1015, 2077, DP2017/1860]; the European Union's Seventh Framework Program (FP7- ChILD-EU 2007-2013) [grant number 305653]; and funding from the patient organizations Respirer c'est Grandir and Belleherbe Association.

\section{Declaration of interest}

The authors have no other relevant affiliations or financial involvement with any organization or entity with a financial interest in or financial conflict with the subject matter or materials discussed in the manuscript apart from those disclosed.

\section{Reviewers Disclosure}

Peer reviewers on this manuscript have no relevant financial relationships or otherwise to disclose.

\section{References}

Papers of special note have been highlighted as either of interest $(\bullet)$ or of considerable interest $(\cdot \bullet)$ to readers.

1. Clement A, Nathan N, Epaud $R$, et al. Interstitial lung diseases in children. Orphanet J Rare Dis. 2010;5:22.

2. Weibel E, Gil J. Structure-function relationships at the alveolar level. West JB, ad. Bioengineering aspect ofthe/ung. New York: Marcel Dekker; 1977. p. 1-81.

3. Kurland G, Deterding RR, Hagood JS, et al. An official American Thoracic Society clinical practice guideline: classification, evalua- tion, and management of childhood interstitial lung disease in infancy. Am J Respir Crit Care Med.
2013;188:376-394.

- A major article on pediatric ILD. 
4. Rice A, Tran-Dang M-A, Bush A, et al. Diffuse lung disease in infancy and childhood: expanding the chILD classification. Histopathology. 2013;63:743-755.

5. American Thoracic Society, European Respiratory Society. American Thoracic Society/European Respiratory Society International Multidisciplinary Consensus Classification of the Idiopathic Interstitial Pneumonias. This joint statement of the American Thoracic Society (ATS), and the European Respiratory Society (ERS) was adopted by the ATS board of directors, June 2001 and by the ERS Executive Committee, June 2001. Am J Respir Crit Care Med. 2002;165(2):277-304.

- A major article on adult ILD.

6. Travis WD, Costabel U, Hansell DM, et al. An official American Thoracic Society/European Respiratory Society statement: update of the international multidisciplinary classification of the idiopathic interstitial pneumonias. Am J Respir Crit Care Med. 2013;188:733-748.

- A major article on adult ILD.

7. Deutsch GH, Young LR, Deterding RR, et al. Diffuse lung disease in young children: application of a novel classification scheme. Am J Respir Crit Care Med. 2007;176:1120-1128.

8. Bush A, Cunningham S, de Blic J, et al. European protocols for the diagnosis and initial treatment of interstitial lung disease in chil- dren. Thorax. 2015;70:1078-1084.

-. A collaborative European work on chILD diagnosis and treatment.

9. Soares JJ, Deutsch GH, Moore PE, et al. Childhood interstitial lung diseases: an 18-year retrospective analysis. Pediatrics. 2013;132:684-691.

10. Nathan N, Thouvenin G, Fauroux B, et al. Interstitial lung disease: physiopathology in the context of lung growth. Paediatric Respir Reviews. 2011;12:216-222.

11. Nogee LM. Interstitial lung disease in newborns. Semin Fetal Neonatal Med. 2017;22(4):227-233.

- A comprehensive review on neonatal ILD.

12. Spagnolo P, Bush A. Interstitial lung disease in children younger than 2 years. Pediatrics. 2016;137(6).

13. Griese M, Seidl E, Hengst M, et al. International management plat- form for children's interstitial lung disease (chlLD-EU). Thorax. 2018 Mar;73(3):231-239.

-. The authors presented the first results of the European database dedicated to chILD. This was a collaborative work of the chILD European consortium.

14. Fan LL, Dishop MK, Galambos C, et al. Diffuse lung disease in biopsied children 2-18 years of age: application of the chILD classification scheme. Ann Am Thorac Soc. 2015;12(10):14981505.

15. Hime NJ, Zurynski Y, Fitzgerald D, et al. Childhood interstitial lung disease: a systematic review. Pediatr Pulmonol. 2015;50:1383-1392.

16. Kuo CS, Young LR. Interstitial lung disease in children. Curr Opin Pediatr. 2014;26:320-327.

17. Vece TJ, Young LR. Update on diffuse lung disease in children Chest. 2016;149:836-845.

18. Dinwiddie R, Sharief $\mathrm{N}$, Crawford O. Idiopathic interstitial pneumo- nitis in children: a national survey in the United Kingdom and Ireland. Pediatr Pulmonol. 2002;34:23-29.

19. Nathan N, Taam RA, Epaud R, et al. A national internet-linked based database for pediatric interstitial lung diseases: the French net- work. Orphanet J Rare Dis. 2012;7:40.

20. Saddi V, Beggs S, Bennetts B, et al. Childhood interstitial lung diseases in immunocompetent children in Australia and New Zealand: a decade's experience. Orphanet $J$ Rare Dis. 2017;12:133.

21. Clement A, de Blic J, Epaud R, et al. Management of children with interstitial lung diseases: the difficult issue of acute exacerbations. Eur Respir J. 2016;48:1559-1563.

- A collaborative work of the chILD European consortium that established criteria to define acute exacerbations in children with chILD, based on a DELPHI process.

22. Bajaj S, Muranjan M, Karande S, et al. Rare disease heralded by pulmonary manifestations: avoiding pitfalls of an "asthma" label. J Postgrad Med. 2017;63:122-127. 
23. Lucaya J, Ducou-le-Pointe H. High-Resolution CT of the lung in children. Pediatric Chest Imaging. Garcia-Pena $P$ and Guillerman P Editors. Springer 2008;111-156.

24. Semple TR, Ashworth MT, Owens CM. Interstitial lung disease in children made easier. . .well, almost. Radiographics. 2017;37:1679- 1703 .

- A very didactic article on chILD imaging.

25. Koh DM, Hansell DM. Computed tomography of diffuse interstitial lung disease in children. Clin Radiol. 2000;55:659667.

26. Guillerman RP. Imaging of childhood interstitial lung disease. Pediatr Allergy Immunol Pulmonol. 2010;23:43-68.

27. Klusmann M, Owens C. HRCT in paediatric diffuse interstitial lung disease-a review for 2009. Pediatr Radiol. 2009;39(Suppl 3):471- 481.

28. Vrielynck S, Mamou-Mani T, Emond S, et al. Diagnostic value of high-resolution CT in the evaluation of chronic infiltrative lung disease in children. AJR Am $J$ Roentgenol. 2008;191:914-920.

29. Dournes G, Grodzki D, Macey J, et al. Quiet submillimeter MR imaging of the lung is feasible with a PETRA sequence at 1. 5 T. Radiology. 2015;276:258-265.

30. Midulla F, Villani A, Merolla $R$, et al. Bronchoalveolar lavage studies in children without parenchymal lung disease: cellular constituents and protein levels. Pediatr Pulmonol. 1995;20:112-118.

31. Fortmann C, Schwerk N, Wetzke $M$, et al. Diagnostic accuracy and therapeutic relevance of thoracoscopic lung biopsies in children. Pediatr Pulmonol. 2018 Jul;53(7):948953.

32. Langston C, Patterson K, Dishop MK, et al. A protocol for the handling of tissue obtained by operative lung biopsy: recommen- dations of the chILD pathology co-operative group. Pediatr Dev Pathol. 2006;9:173-180.

33. Edwards V, Cutz E, Viero S, et al. Ultrastructure of lamellar bodies in congenital surfactant deficiency. Ultrastruct Pathol. 2005;29:503-509.

34. Canakis A-M, Cutz E, Manson D, et al. Pulmonary interstitial glyco- genosis: a new variant of neonatal interstitial lung disease. Am J Respir Crit Care Med. 2002;165:1557-1565.

35. Fink JN, Ortega HG, Reynolds HY, et al. Needs and opportunities for research in hypersensitivity pneumonitis. Am J Respir Crit Care Med. 2005;171:792-798.

36. Venkatesh P, Wild L. Hypersensitivity pneumonitis in children: clinical features, diagnosis, and treatment. Paediatr Drugs. 2005;7:235-244.

37. Charpin D, Baden R, Bex V, et al. Environmental home inspection services in Western Europe. Environ Health Prev Med. 2011;16:73-79.

38. Vasakova M, Morell F, Walsh S, et al. Hypersensitivity pneumonitis: perspectives in diagnosis and management. Am J Respir Crit Care Med. 2017;196:680-689.

- A comprehensive article on HP in adults.

39. Stauffer Ettlin M, Pache J-C, Renevey F, et al. Bird breeder's disease: a rare diagnosis in young children. Eur $\mathrm{J}$ Pediatr. 2006;165:55-61.

40. Nacar N, Kiper N, Yalcin E, et al. Hypersensitivity pneumonitis in children: pigeon breeder's disease. Ann Trop Paediatr. 2004;24:349-355.

41. Yoon HM, Lee E, Lee JS, et al. Humidifier disinfectantassociated children's interstitial lung disease: computed tomographic features, histopathologic correlation and comparison between survivors and non-survivors. Eur Radiol. 2016;26:235-243.

42. Kim KW, Ahn K, Yang $\mathrm{HJ}$, et al. Humidifier disinfectantassociated children's interstitial lung disease. Am J Respir Crit Care Med. 2014;189:48-56.

43. Magon $P$. Reversible lung disease due to abundant use of talcum powder. Indian J Pediatr. 2012;79:1383.

44. Dell S, Cernelc-Kohan M, Hagood JS. Diffuse and interstitial lung disease and childhood rheumatologic disorders. Curr Opin Rheumatol. 2012;24:530-540.

- A review on rheumatologic related ILD.

45. Tarantino G, Esposito S, Andreozzi L, et al. Lung involvement in children with hereditary autoinflammatory disorders. Int J Mol Sci.
2016;17(12).

46. Calender A, Rollat Farnier PA, Buisson A, et al. Whole exome sequencing in three families segregating a pediatric case of sarcoi- dosis. BMC Med Genomics. 2018;11:23. 
47. Nathan N, Marcelo P, Houdouin V, et al. Lung sarcoidosis in chil- dren: update on disease expression and management. Thorax. 2015;70:537-542.

- A recent update and large experience on pediatric sarcoidosis.

48. Gülhan B, Ozçelik U, Gürakan F, et al. Different features of lung involvement in Niemann-Pick disease and Gaucher disease. Respir Med. 2012;106:1278-1285.

49. Hengst M, Naehrlich L, Mahavadi $P$, et al. HermanskyPudlak syn- drome type 2 manifests with fibrosing lung disease early in child- hood. Orphanet $J$ Rare Dis. 2018;13:42.

50. Taytard J, Nathan N, de Blic J, et al. New insights into pediatric idiopathic pulmonary hemosiderosis: the French RespiRare(囚) cohort. Orphanet J Rare Dis. 2013;8:161.

- A recent update and large experience on pediatric sarcoidosis.

51. Giovannini-Chami L, Hadchouel A, Nathan N, et al. Idiopathic eosi- nophilic pneumonia in children: the French experience. Orphanet J Rare Dis. 2014;9:28.

- A recent update and large experience on idiopathic eosinophi- lic pneumonia.

52. Epaud R, Fauroux B, Boule M, et al. Diseases of the pulmonary lymphatic system in children. Rev Pneumol Clin. 2003:59:7-15.

53. Young LR, Deutsch GH, Bokulic RE, et al. A mutation in TTF1/NKX2.1 is associated with familial neuroendocrine cell hyperplasia of infancy. Chest. 2013;144:1199-1206.

54. Nevel RJ, Garnett ET, Worrell JA, et al. Persistent lung disease in adults with NKX2.1 mutation and familial neuroendocrine cell hyperplasia of infancy. Ann Am Thorac Soc. 2016;13:1299-1304.

55. Jiramethee N, Erasmus D, Nogee L, et al. Pulmonary neuroendo- crine cell hyperplasia associated with surfactant protein C gene mutation. Case Rep Pulmonol. 2017;2017:9541419

56. Rauch D, Wetzke M, Reu S, et al. Persistent tachypnea of infancy. Usual Aberrant Am J Respir Crit Care Med. 2016;193:438-447.

57. Houin PR, Deterding RR, Young LR. Exacerbations in neuroendo- crine cell hyperplasia of infancy are characterized by increased air trapping. Pediatr Pulmonol. 2016;51:E9-12.

58. Young LR, Brody AS, Inge TH, et al. Neuroendocrine cell distribution and frequency distinguish neuroendocrine cell hyperplasia of infancy from other pulmonary disorders. Chest. 2011;139:1060-1071.

59. Yancheva SG, Velani A, Rice A, et al. Bombesin staining in neuroen- docrine cell hyperplasia of infancy (NEHI) and other childhood interstitial lung diseases (chILD). Histopathology. 2015 Oct;67 (4):501-508.

60. Cutz E, Chami R, Dell S, et al. Pulmonary interstitial glycogenosis associated with a spectrum of neonatal pulmonary disorders. Hum Pathol. 2017;68:154-165.

61. Langston C, Dishop MK. Diffuse lung disease in infancy: a proposed classification applied to 259 diagnostic biopsies. Pediatr Dev Pathol. 2009:12:421-437.

62. Szafranski P, Coban-Akdemir ZH, Rupps R, et al. Phenotypic expan- sion of TBX4 mutations to include acinar dysplasia of the lungs. Am J Med Genet A. 2016;170:24402444.

63. Barnett CP, Nataren NJ, Klingler-Hoffmann M, et al. Ectrodactyly and lethal pulmonary acinar dysplasia associated with homozygous FGFR2 mutations identified by exome sequencing. Hum Mutat. 2016;37:955-963.

64. Stankiewicz P, Sen $P$, Bhatt SS, et al. Genomic and genic deletions of the FOX gene cluster on 16q24.1 and inactivating mutations of FOXF1 cause alveolar capillary dysplasia and other malformations. Am J Hum Genet. 2009;84:780-791.

65. Szafranski $P$, Dharmadhikari AV, Wambach JA, et al. Two deletions overlapping a distant FOXF1 enhancer unravel the role of IncRNA LINC01081 in etiology of alveolar capillary dysplasia with misalign- ment of pulmonary veins. Am J Med
Genet A. 2014;164A:2013-2019.

66. Slot E, Edel G, Cutz E, et al. Alveolar capillary dysplasia with mis- alignment of the pulmonary veins: clinical, histological, and genetic aspects. Pulm Circ. 2018;8:2045894018795143.

67. Lange M, Kasper B, Bohring $A$, et al. 47 patients with FLNA asso- ciated periventricular nodular heterotopia. Orphanet $\mathrm{J}$ Rare Dis. 2015;10:134. 
68. Shelmerdine SC, Semple T, Wallis C, et al. Filamin A (FLNA) muta- tion-A newcomer to the childhood interstitial lung disease (ChILD) classification. Pediatr Pulmonol. 2017;52:1306-1315.

69. Nathan N, Borensztajn K, Clement A. Genetic causes and clinical management of pediatric interstitial lung diseases. Curr Opin Pulm Med. 2018;24(3):253-259.

70. Gupta A, Zheng SL. Genetic disorders of surfactant protein dysfunc- tion: when to consider and how to investigate. Arch. Dis. Child. 2017;102:84-90.

- The authors reviewed the current knowledge on surfactant disorders in children, mainly in relation with SP-B, SP-C, $A B C A 3$ and NKX2-1 deficiencies: clinical presentation, investigations, management.

71. Litao MKS, Hayes D, Chiwane S, et al. A novel surfactant protein $\mathrm{C}$ gene mutation associated with progressive respiratory failure in infancy. Pediatr Pulmonol. 2017;52:57-68.

72. Delestrain C, Simon S, Aissat A, et al. Deciphering the mechanism of Q145H SFTPC mutation unmasks a splicing defect and explains the severity of the phenotype. Eur $\mathrm{J}$ Hum Genet. 2017;25:779-782

73. Salerno T, Peca D, Menchini L, et al. Surfactant Protein Cassociated interstitial lung disease; three different phenotypes of the same SFTPC mutation. Ital J Pediatr. 2016;42:23.

- An article that reinforces the knowledge that the most frequent mutation of SFTPC, 173T, is associated with a highly variable phenotype.

74. Hawkins A, Guttentag SH, Deterding R, et al. A non-BRICHOS SFTPC mutant (SP-Cl73T) linked to interstitial lung disease promotes a late block in macroautophagy disrupting cellular proteostasis and mito- phagy. Am $\mathrm{J}$ Physiol Lung Cell Mol Physiol. 2015;308:L33-47.

75. Avital A, Hevroni A, Godfrey S, et al. Natural history of five children with surfactant protein $\mathrm{C}$ mutations and interstitial lung disease. Pediatr Pulmonol. 2014;49:1097-1105.

76. Abou Taam R, Jaubert $F$, Emond $S$, et al. Familial interstitial disease with 173T mutation: A mid- and long-term study. Pediatr Pulmonol. 2009;44:167-175

77. Shulenin S, Nogee LM, Annilo T, et al. ABCA3 gene mutations in newborns with fatal surfactant deficiency. $\mathrm{N}$ Engl $\mathrm{J}$ Med. 2004:350:1296-1303.

78. Zhou W, Zhuang Y, Sun J, et al. Variants of the ABCA3 gene might contribute to susceptibility to interstitial lung diseases in the Chinese population. Sci Rep. 2017;7:4097.

79. Campo I, Zorzetto $M$, Mariani $F$, et al. A large kindred of pulmonary fibrosis associated with a novel ABCA3 gene variant. Respir Res. 2014;15:43.

80. Kröner C, Wittmann T, Reu S, et al. Lung disease caused by ABCA3 mutations. Thorax. 2017;72:213-220.

- The authors reported a large population of patients with ABCA3 mutations, with information on phenotypic expression and management strategies.

81. Thavagnanam S, Cutz E, Manson D, et al. Variable clinical outcome of $A B C A 3$ deficiency in two siblings. Pediatr Pulmonol. 2013;48:1035-1038.

82. Wambach JA, Wegner DJ, Depass $K$, et al. Single ABCA3 mutations increase risk for neonatal respiratory distress syndrome. Pediatrics. 2012;130:e1575-1582.

83. Devriendt K, Vanhole C, Matthijs G, et al. Deletion of thyroid transcription factor-1 gene in an infant with neonatal thyroid dysfunction and respiratory failure. $N$ Engl $J$ Med. 1998;338:1317-1318.

84. Hamvas A, Deterding RR, Wert SE, et al. Heterogeneous pulmonary phenotypes associated with mutations in the thyroid transcription factor gene NKX2-1. Chest. 2013:144:794-804.

85. Nattes E, Lejeune S, Carsin A, et al. Heterogeneity of lung disease associated with NK2 homeobox 1 mutations. Respir Med. 2017;129:16-23.

- The authors presented a large cohort of patients with NKX2-1 mutations with description of disease expression.

86. Nogee LM, de Mello DE, Dehner LP, et al. Brief report: deficiency of pulmonary surfactant protein $B$ in congenital alveolar proteinosis. N Engl J Med. 1993;328:406-410.
87. Nogee LM, Garnier G, Dietz HC, et al. A mutation in the surfactant protein $B$ gene responsible for fatal neonatal respiratory disease in multiple kindreds. $\mathrm{J}$ Clin Invest. 1994;93:1860-1863.

88. Hoover RR, Floros J. Organization of the human SP-A and SP-D loci at 10q22-q23. Physical and radiation hybrid mapping reveal gene order and orientation. Am J Respir Mol Biology. 1998;18:353-362.

89. Silveyra P, Floros J. Genetic complexity of the human surfactant- associated proteins SP-A1 and SP-A2. Gene. 2013:531:126-132.

90. Wang Y, Kuan PJ, Xing C, et al. Genetic defects in surfactant protein A2 are associated with pulmonary fibrosis and lung cancer. Am J Hum Genetics. 2009;84:52-59.

91. Coghlan MA, Shifren A, Huang $H J$, et al. Sequencing of idiopathic pulmonary fibrosis-related genes reveals independent single gene associations. BMJ Open Respir Res. 2014;1:e000057.

92. van Moorsel CHM, Ten Klooster L, van Oosterhout MFM, et al. SFTPA2 mutations in familial and sporadic idiopathic interstitial pneumonia. Am J Respir Crit Care Med. 2015:192:1249-1252.

93. Nathan N, Giraud V, Picard C, et al. Germline SFTPA1 mutation in familial idiopathic interstitial pneumonia and lung cancer. Hum Mol Genet. 2016;25:1457-1467.

- In this article, we reported the first description of a pathogenic SFTPA1 mutation associated with various forms of interstitial pneumonia or lung fibrosis and adenocarcinoma of the lung. The mutation was found in a large family that included both children and adults patients.

94. Hadchouel A, Wieland T, Griese M, et al. Biallelic mutations of methionyl-tRNA synthetase cause a specific type of pulmonary alveolar proteinosis prevalent on réunion island. Am J Hum Genet. 2015;96:826-831.

95. Hildebrandt J, Yalcin E, Bresser H-G, et al. Characterization of CSF2RA mutation related juvenile pulmonary alveolar proteinosis. Orphanet J Rare Dis. 2014;9:171.

96. Suzuki T, Maranda B, Sakagami T, et al. Hereditary pulmonary alveolar proteinosis caused by recessive CSF2RB mutations. Eur Respir J. 2011;37:201-204.

97. Tarantino G, Esposito S, Andreozzi L, et al. Lung involvement in children with hereditary autoinflammatory disorders. Int J Mol Sci. 2016; 17.

- The article was a review on autoinflammatory diseases with a lung involvement, including SAVI (due to TMEM173 mutations), and COPA syndrome.

98. Melki I, Rose Y, Uggenti C, et al. Disease-associated mutations iden- tify a novel region in human STING necessary for the control of type I interferon signaling. J Allergy Clin Immunol. 2017;140(543-552):e5.

99. Picard C, Thouvenin G, Kannengiesser C, et al. Severe pulmonary fibrosis as the first manifestation of interferonopathy (TMEM173 mutation). Chest. 2016:150:e65-71.

100. Jeremiah N, Neven B, Gentili M, et al. Inherited STINGactivating mutation underlies a familial inflammatory syndrome with lupus- like manifestations. J Clin Invest 2014; 124:5516-5520.

101. Liu $Y$, Jesus AA, Marrero $B$, et al. Activated STING in a vascular and pulmonary syndrome. $N$ Engl $J$ Med. 2014;371:507-518.

102. Watkin LB, Jessen B, Wiszniewski W, et al. COPA mutations impair ER-Golgi transport and cause hereditary autoimmunemediated lung disease and arthritis. Nat Genet. 2015;47:654660.

-. The authors described a new autoinflammatory disorder due to COPA mutations. All of the described patients presented a lung involvement with ILD or hemosiderosis.

103. Jensson BO, Hansdottir S, Arnadottir GA, et al. COPA syndrome in an Icelandic family caused by a recurrent missense mutation in COPA. BMC Med Genet. 2017;18:129.

104. Vece TJ, Watkin LB, Nicholas S, et al. Copa syndrome: a novel autosomal dominant immune dysregulatory disease. J Clin Immunol. 2016:36:377-387.

105. Nathan N, Borie R, Kannengiesser C, et al. Genetic testing in idio- pathic interstitial pneumonia. Eur Respir $J$ 
2015;46:PA4849.

106. Brown AW, Kaya $H$, Nathan SD. Lung transplantation in IIP: A review. Respirology. 2016 Oct;21(7):1173-1184.

107. Eldridge WB, Zhang Q, Faro A, et al. Outcomes of lung transplanta- tion for infants and children with genetic disorders of surfactant metabolism. J Pediatr. 2017;184(157-164):e2. 